\title{
Validation of the Western Ontario and McMaster Universities Osteoarthritis Index in Patients Having Undergone Ankle Fracture Surgery
}

\author{
Ville T. Ponkilainen, $\mathrm{BM}^{1}$, Arja H. Häkkinen, $\mathrm{PhD}^{2,3}$, Mikko M. Uimonen, $\mathrm{BM}^{1}$, \\ Erkki Tukiainen, MD, $\mathrm{PhD}^{4}$, Henrik Sandelin, $\mathrm{MD}^{5}$, Jussi P. Repo, MD, $\mathrm{PhD}^{1}$ \\ ${ }^{1}$ Resident, Department of Surgery, Central Finland Central Hospital, Jyväskylä, Finland \\ ${ }^{2}$ Professor, Health Sciences, Faculty of Sport and Health Sciences, University of Jyväskylä, Jyväskylä, Finland \\ ${ }^{3}$ Professor, Department of Physical Medicine, Central Finland Health Care District, Jyväskylä, Finland \\ ${ }^{4}$ Professor, Department of Plastic Surgery, Central Hospital of Helsinki University, HUS, Helsinki, Finland \\ ${ }^{5}$ Orthopaedic Surgeon, Department of Orthopaedics and Traumatology, HUS Helsinki University Hospital and University of Helsinki, Helsinki, Finland
}

\section{A R T I C L E I N F O}

Level of Clinical Evidence: 3

Keywords:

ankle

LEFS

psychometrics

reliability validity

VAS-FA

WOMAC

\begin{abstract}
A B S T R A C T
The Western Ontario and McMaster Universities Osteoarthritis Index (WOMAC) is a patient-reported outcome measure (PROM) that is widely used to evaluate the pain, stiffness, and physical function of patients with osteoarthritis of the hip and knee. Although the WOMAC has also been used for patients after foot and ankle surgery, it has not been validated for this purpose. A total of 130 patients with surgically treated ankle fractures completed the WOMAC, Visual Analogue Scale Foot and Ankle (VAS FA), Lower Extremity Functional Scale (LEFS), 15D Health-Related Quality-of-Life Questionnaire (15D), and Visual Analog Scale for General Health (VAS general health) after foot and ankle surgery. The structural validity of the WOMAC was assessed by using Cronbach's $\alpha$, and convergent validity was tested between the WOMAC and reference outcome measures. Cronbach's $\alpha$ for the index score was 0.98 and $0.95,0.86$, and 0.98 for the Pain, Stiffness, and Physical Function subscales, respectively. The Spearman correlation coefficients were $-0.84,-0.74,-0.58$, and 0.55 for the VAS-FA, LEFS, 15D, and VAS general health, respectively. The relationships with the VAS-FA, LEFS, 15D, and VAS general health were strong. All relationships were statistically significant $(p<.001)$. The WOMAC provides valid scores for assessing pain, stiffness, and physical function in patients having undergone ankle fracture surgery.
\end{abstract}

(c) 2019 by the American College of Foot and Ankle Surgeons. All rights reserved.
Patient-reported outcome measures (PROMs) are useful tools in evaluating outcomes after surgical interventions (1). The use of PROMs in clinical practice and scientific studies has gained increased interest in recent years, because these measures provide a means to evaluate outcomes from the patients' perspective $(2,3)$. The benefits of using PROMs are evaluation of the patients' functional status, the differences between patients, the effectiveness of treatment, and the ability to perform benchmarking (4).

There is a broad variety of PROMs used among foot and ankle patients, with notable differences in the validity and reliability of these tools (1). There is no consensus on which PROMs to use for any individual procedures in the treatment of foot and ankle pathologies $(5,6)$. The US Food and Drug Administration created guidelines for the proper development and validation of PROMs that are intended for medical

Financial Disclosure: None reported.

Conflicts of Interest: None reported.

Address correspondence to: Ville T. Ponkilainen, BM, Department of Surgery, Central Finland Central Hospital, Keskussairaalantie 19, 40620 Jyväskylä, Finland.

E-mail address: ponkilainen.ville.t@student.uta.fi (V.T. Ponkilainen). use $(4,7,8)$. The US Food and Drug Administration guidelines emphasize that PROMs should always be reviewed for reliability, validity, ability to detect change, and interpretability before application in clinical or research use (4). The measurement properties should be assessed for the current study population and study design (4). These guidelines are important to make the field of outcome measures consistent and clear $(9,10)$.

The Western Ontario and McMaster Universities Osteoarthritis Index (WOMAC) is a PROM that is generally used for evaluating physical disability and symptoms in patients with osteoarthritis of the hip and knee $(11,12)$. There are 2 versions of the WOMAC scale. One version uses a Likert scale (scores from 1 to 5 , categorical) and the other uses a Visual Analog Scale (VAS) (scores from 0 to 100 , continuous) (13). The VAS scaled version has been found to be valid, reliable, responsive, and disease specific for hip and knee osteoarthritis, rheumatoid arthritis, and fibromyalgia $(11,12,14,15)$.

The validity and reliability of the WOMAC questionnaire have not been tested for the assessment of outcomes after ankle surgery. The aim of this study was to validate the VAS version of the WOMAC questionnaire for patients having undergone ankle fracture surgery. 


\section{Patients and Methods}

Patients were identified from a database into which patients had been previously prospectively entered or searched from hospital electronic databases by using National Institute for Health and Welfare procedure codes (NHJ10 [ankle fracture osteosynthesis]; NHU20 [removal of implants from foot or ankle]). The inclusion criteria were full understanding of written Finnish, age $\geq 18$ years, and previous foot and ankle surgery. The exclusion criteria were isolated foot fractures and surgery as a result of infection, tumor, or osteoarthritis.

The patients were approached in a cross-sectional manner via mail. Patients who agreed to participate completed the WOMAC, the Lower Extremity Functional Scale (LEFS), the Visual Analogue Scale Foot and Ankle (VAS-FA), and the 15D generic Health-Related Quality of Life (HRQoL) (15D) instruments. In addition, patients completed questions about general health state, physical activity [Kasari FIT index (16)], and a VAS of general health. The study protocol was approved by the regional ethics committee of the hospital district.

\section{Instruments}

\section{WOMAC}

The WOMAC (version 3.0) is a VAS scaled 24-item instrument consisting of 3 subscales: Pain, Stiffness, and Physical Function $(11,12,17)$. The Pain subscale consists of 5 items, the Stiffness subscale consists of 2 items, and the Physical Function subscale consists of 17 items (17). Higher scores in the WOMAC scale and in its subscales indicate greater degree of physical disability and more symptoms, whereas lower scores indicate better physical performance and fewer symptoms (17). Each subscale has been found to fit well in the Rasch model, and the Pain and Physical Function subscales have shown unidimensional construct in factor analyses (17). Cronbach's $\alpha$ (internal consistency) values were $.82, .80$, and .95 for the Pain, Stiffness, and Physical Function subscales, respectively (17). The WOMAC has been translated, and both versions have been validated in the Finnish language $(18,19)$. The modified instructions of the questionnaire asked for the patient's view about his or her foot, ankle, or both in completing the survey.

\section{VAS-FA}

The VAS-FA is a foot and ankle-specific PROM that has been validated for foot and ankle patients to assess function, pain, and other complaints $(20-22)$. The scale contains 20 items on a VAS $(0-100 \mathrm{~mm}$, worst to best) and yields a score between 0 and 100 (23). The items are divided into 3 modules as follows: Pain (4 items), Function (11 items), and Other Complaints ( 5 items) (23). Cronbach's $\alpha$ values for the subscales of the questionnaire have been evaluated to be .94 for Function, .91 for Pain, and .81 for Other Complaints, thus indicating high internal consistency (22). The VAS-FA has very high intraclass correlation coefficients for total score (0.97) and its different subscales (0.95-0.97), indicating high relative reliability (22). The VAS-FA has been validated in the Finnish language (22).

\section{LEFS}

The LEFS is a patient-reported outcome instrument assessing the function of patients after foot and ankle surgery $(24,25)$. It consists of 20 items with 5 response categories. The LEFS is scored from 0 to 4 points, with higher scores indicating better function and lower scores indicating worse function (25). The LEFS instrument has been shown to be reliable and to have high construct validity and sensitivity to detect change in assessing the foot and ankle function (25). Cronbach's $\alpha$ value for the LEFS is .96, indicating high internal consistency (25). The Finnish version of the LEFS has comparable validity and reliability with the original version of the LEFS (26).

\section{$15 D$}

The $15 \mathrm{D}$ is a generic, self-administered HRQoL instrument consisting of 15 subscales measuring distinct dimensions related to HRQoL (27):
Moving, Seeing, Hearing, Breathing, Sleeping, Eating, Speech, Excretion, Usual Activities, Mental Function, Discomfort and Symptoms, Depression, Distress, Vitality, and Sexual Activity. All items have 5 response categories from 1 (representing the best state) to 5 (representing the worst state) for each dimension. The 15D index is calculated from all subscale scores. The index score varies from 0 to 1 , representing the worst to the best imaginable state of HRQoL. The 15D has been compared with other generic HRQoL instruments in previous international studies, in which the 15D has been shown to yield comparable results to the reference measures $(27-30)$.

\section{Statistical Methods}

Clinical and sociodemographic data are presented as mean \pm standard deviation (SD) values, medians with interquartile ranges (IQRs), 95\% confidence intervals (CIs), or counts with percentages. Distribution of the WOMAC score was assessed. Scale targeting and coverage were investigated by calculating the percentage of minimum and maximum points. A floor or ceiling effect was considered to be confirmed when $15 \%$ of patients scored the minimum or maximum points (30). The authors hypothesized that the WOMAC would not reach the floor or ceiling values. Cronbach's $\alpha$ (internal consistency) was used to evaluate the structural validity of the WOMAC instrument (31). The authors hypothesized that the internal consistency would be not less than .70 (32).

The Spearman correlation coefficients of the WOMAC and reference instruments were calculated to assess convergent validity of the WOMAC instrument. The correlations were represented according to the rule of thumb for Interpreting the size of a correlation coefficient (33), namely as negligible (0.00-0.29), low (0.30-0.49), moderate (0.50-0.69), high (0.70-0.89), and very high $(0.90-1.00)$ correlation. Linearity between the WOMAC and reference outcomes (the LEFS, the VAS-FA, and the 15D mean score) was assessed. Further, linear regression analyses were used to identify the appropriate predictors of the WOMAC standardized by age, postoperative body mass index (BMI), and sex. The regression coefficient $\beta$ value indicates how strongly each predictor variable influences the criterion variable (the mean LEFS, VAS-FA, and 15D scores). The $\beta$ value was measured in units of SD. Cohen reference values of $0.1,0.3$, and 0.5 refer to small, moderate, and strong correlations, respectively. The authors hypothesized that there would be a strong correlation between the WOMAC and the reference instruments.

Patients with complete data were added to the analyses. Analyses were performed by using $\mathrm{R}$ (version 1.1.453) statistics software. Results are reported according to the Strengthening the Reporting of Observational Studies in Epidemiology (STROBE) statement (34) and the COnsensus-based Standards for the selection of health status Measurement Instruments (COSMIN) (35).

\section{Results}

Of the total of 212 recruited patients, $130(61 \%)$ met the inclusion criteria and were included in the analysis (Table 1). The mean follow-up time was 4 years (range 1 month to 14 years) after the last operation. Of the 130 included patients, 49 (38\%) patients had lateral malleolus fracture, $37(28 \%)$ had trimalleolar fracture, 31 (24\%) had medial malleolus fracture, and 13 (10\%) had other fractures. Fractures were either closed $(n=119)$ or open $(n=11)$. Surgery was performed with plates and screws $(n=116[89 \%])$, screws $(n=5[4 \%])$, soft tissue flap $(n=5[4 \%])$, external fixation $(n=1[1 \%])$, tibial nail $(n=1[1 \%])$, or multiple techniques $(n=2[2 \%])$.

The median (range) of the WOMAC index score was 8.0 (0 to 85.9) points (Figs. 1 and 2). Minimum points were observed for 4 patients. No patients scored maximum points. Floor or ceiling 
Table 1

Sociodemographic characteristics of the participants $(\mathrm{N}=130)$

\begin{tabular}{lc}
\hline Characteristic & \\
\hline Age, mean \pm SD, $\mathrm{y}$ & $56 \pm 17$ \\
Female, $\mathrm{n}(\%)$ & $74(57)$ \\
Marital status n (\%) & \\
$\quad$ Married & $68(52)$ \\
Widow & $11(8)$ \\
Divorced & $24(18)$ \\
$\quad$ Unmarried & $26(20)$ \\
In working life, $\mathrm{n}(\%)$ & $55(42)$ \\
Smokers, $\mathrm{n}(\%)$ & $22(17)$ \\
Diabetes, $\mathrm{n}(\%)$ & $11(8)$ \\
Atherosclerosis, $\mathrm{n}(\%)$ & $1(1)$ \\
BMI, mean $\pm \mathrm{kg} / \mathrm{m}^{2}$ & $27 \pm 5$ \\
Operations, $\mathrm{n}(\%)$ & \\
1 & $85(65)$ \\
2 & $17(13)$ \\
3 & $11(8)$ \\
4 & $4(3)$ \\
5 & $2(2)$ \\
7 & $2(2)$ \\
11 & $1(1)$ \\
\hline
\end{tabular}

Abbreviation: $\mathrm{BMI}=$ body mass index. effects (threshold of 15\%) were not observed. The score distribution did not follow a normal distribution, because most patients' scores concentrated around the lower points, indicating a higher level of function and a lower level of stiffness. Cronbach's $\alpha$ for the index score was .98 and $.95, .86$, and .98 for the Pain, Stiffness, and Physical Function subscales, respectively (Table 2).

The Spearman correlation coefficients between the subscales of the WOMAC and the reference outcome measures (VAS-FA, LEFS, 15D, and VAS general health) are presented in Table 3. The correlation between the WOMAC Stiffness subscale and all reference outcome measures was lower $(r=0.45-0.75)$ than the correlation between other subscales. The correlations between the WOMAC and the VAS-FA $(r=-0.84)$ and the LEFS $(r=-0.74)$ were high and moderate between the WOMAC and the 15D $(r=-0.58)$, respectively (Figs. $2-4)$. The correlation with VAS general health ranged from low to moderate $(r=0.46-0.62 ; p<.001)$. The Spearman correlation coefficient between the WOMAC and the VAS-FA Pain subscales was $-0.78(p<.001)$ (Fig. 5), and that between the WOMAC Physical function and VAS-FA Function subscales was $-0.83(p<.001)$ (Fig. 6), representing strong negative correlations. The correlation between the WOMAC and time from surgery was -0.21 $(p=.008)$ (Fig. 7).

The age-, BMI-, and sex-standardized relationships between WOMAC and reference outcome measures (VAS-FA, LEFS, 15D, and VAS general health) were assessed (Fig. 8). The relationships were strong with VAS-FA,

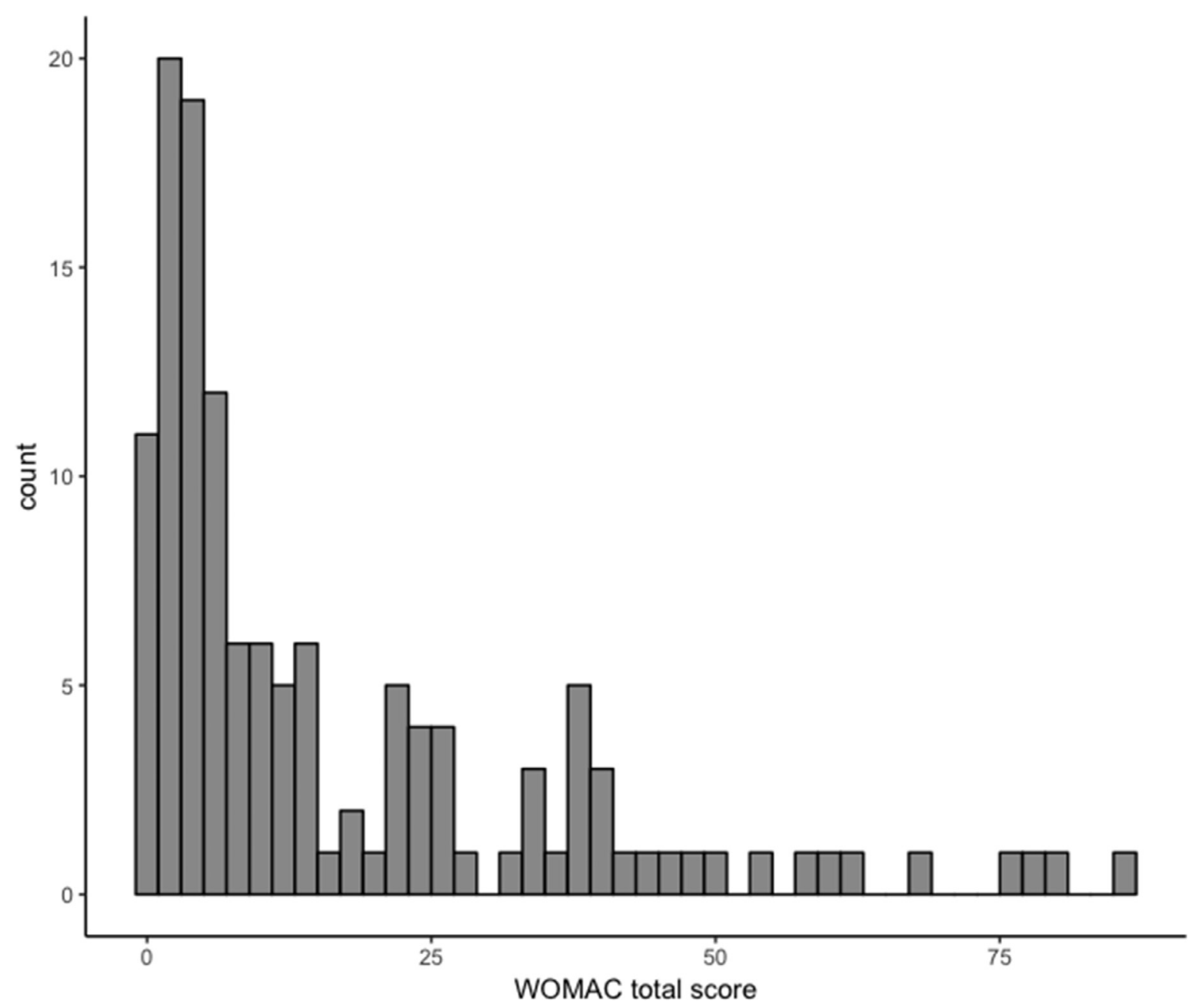

Fig. 1. Distribution of the WOMAC index score in patients having undergone ankle fracture surgery. 


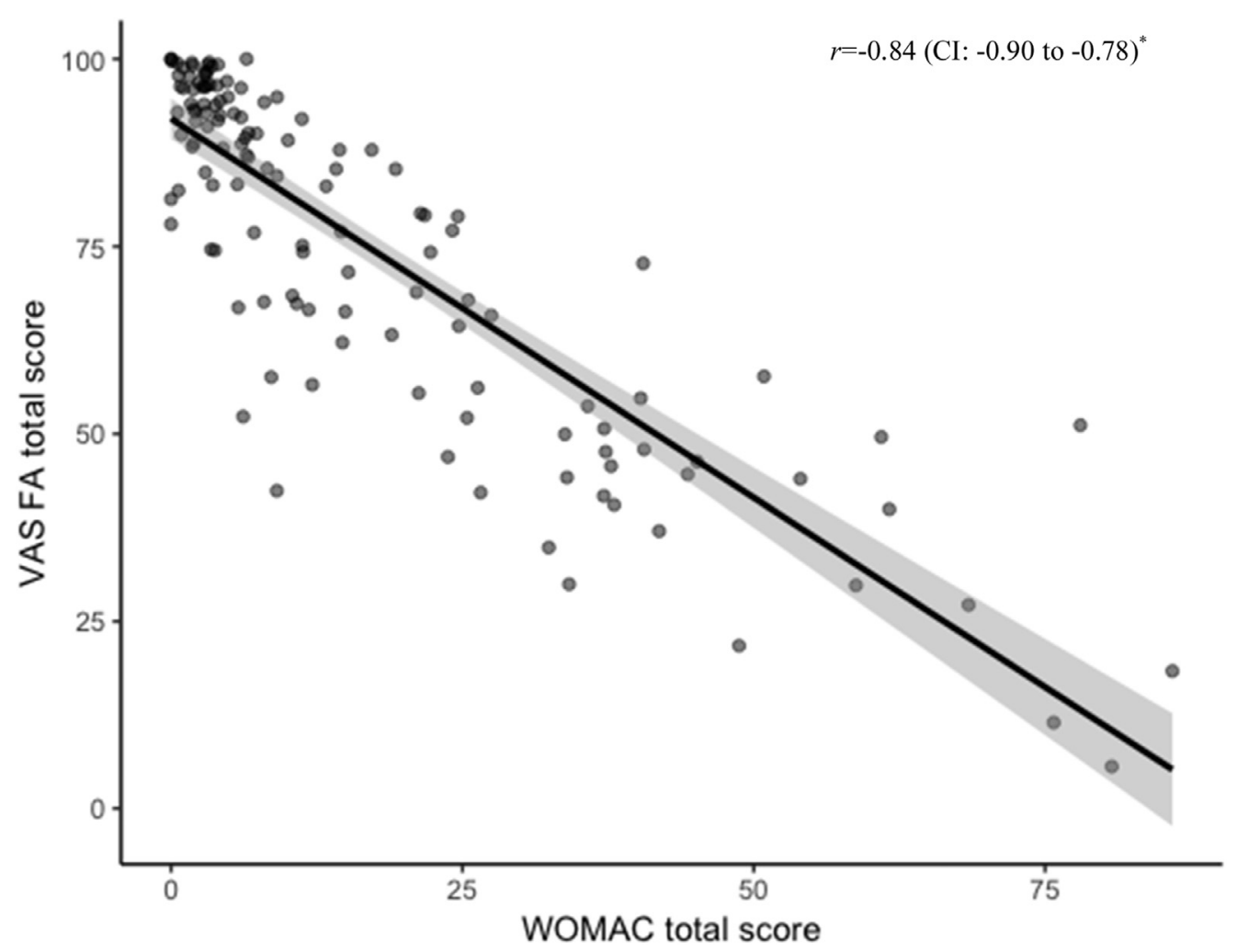

Fig. 2. Correlation between the WOMAC total score and the VAS-FA score.

*Spearman correlation coefficient $(95 \% \mathrm{CI})$.

LEFS, and 15D and moderate with VAS general health. All relationships were statistically significant $(p<.001)$.

\section{Discussion}

The WOMAC has been previously used to evaluate outcomes after the treatment of foot and ankle osteoarthritis and has been used to validate other PROMs for ankle osteoarthritis $(36,37)$. When different lower-extremity PROMs (including the WOMAC and the LEFS) were used among patients with ankle osteoarthritis, none of the PROMs captured all patient concerns and a lack of consensus for which PROM to use remains (37). Likewise, there is no consensus on which foot and

Table 2

Instrument scores of the participants $(\mathrm{N}=130)$

\begin{tabular}{lc}
\hline Instrument Score & \\
\hline General health VAS, median (IQR) & $16(39)$ \\
Physical activity, FIT index, mean \pm SD & $42 \pm 22$ \\
WOMAC, median (IQR) & \\
Index score & $8(22)$ \\
Pain & $6(16)$ \\
Stiffness & $8(33)$ \\
Physical Function & $6(13)$ \\
VAS-FA, median (IQR) & \\
Index score & $83(37)$ \\
Pain & $81(38)$ \\
Function & $85(37)$ \\
Other Complaints & $82(36)$ \\
LEFS score, median (IQR) & $70(18)$ \\
\hline
\end{tabular}

Abbreviations: LEFS $=$ Lower Extremity Functional Scale; VAS$\mathrm{FA}=$ Visual Analogue Scale Foot and Ankle; WOMAC = Western Ontario and McMaster Universities Osteoarthritis Index. ankle-specific PROMs to use among patients having undergone ankle fracture surgery $(1,6,38,39)$. Nevertheless, the WOMAC has been widely used in the field of foot and ankle surgery (40-43), although it has not been previously validated for this purpose. The results of our study indicate that the WOMAC provides valid and reliable scores among patients after ankle fracture surgery.

The Likert scaled WOMAC for knee and hip osteoarthritis was first introduced by Bellamy et al $(11,12)$. The Likert scaled WOMAC has shown good validity and reliability in patients with hip, knee, and ankle osteoarthritis $(11,12,44)$. When the construct validity of the WOMAC questionnaire was evaluated by using Rasch analysis (45), not all items of the Likert-scale version fit the Rasch model and the questionnaire was revised to fulfill the pertinent criteria for the Rasch model (14,46-49). The VAS scaled WOMAC (version 3.0) was first validated by Kersten et al (17) to patch the poor fit of some items in the Likert scaled version. Each subscale of the VAS scaled WOMAC fits well in the Rasch model, and the Pain and Physical Function subscales show unidimensional construct in factor analysis (17). The Stiffness subscale has shown

Table 3

Spearman correlation coefficients between the WOMAC, its subscales, and reference measures

\begin{tabular}{llllc}
\hline & VAS FA & LEFS & $15 \mathrm{D}$ & General Health \\
\hline WOMAC & $-0.84^{*}$ & $-0.74^{*}$ & $-0.58^{*}$ & $0.55^{*}$ \\
Pain subscale & $-0.77^{*}$ & $-0.69^{*}$ & $-0.68^{*}$ & $0.49^{*}$ \\
Stiffness subscale & $-0.75^{*}$ & $-0.66^{*}$ & $-0.45^{*}$ & $0.46^{*}$ \\
Physical Function subscale & $-0.83^{*}$ & $-0.78^{*}$ & $-0.62^{*}$ & $0.62^{*}$ \\
\hline
\end{tabular}

Abbreviations: LEFS = Lower Extremity Functional Scale; VAS-FA = Visual Analogue Scale Foot and Ankle; WOMAC = Western Ontario and McMaster Universities Osteoarthritis Index.

${ }^{*} p<.001$. 


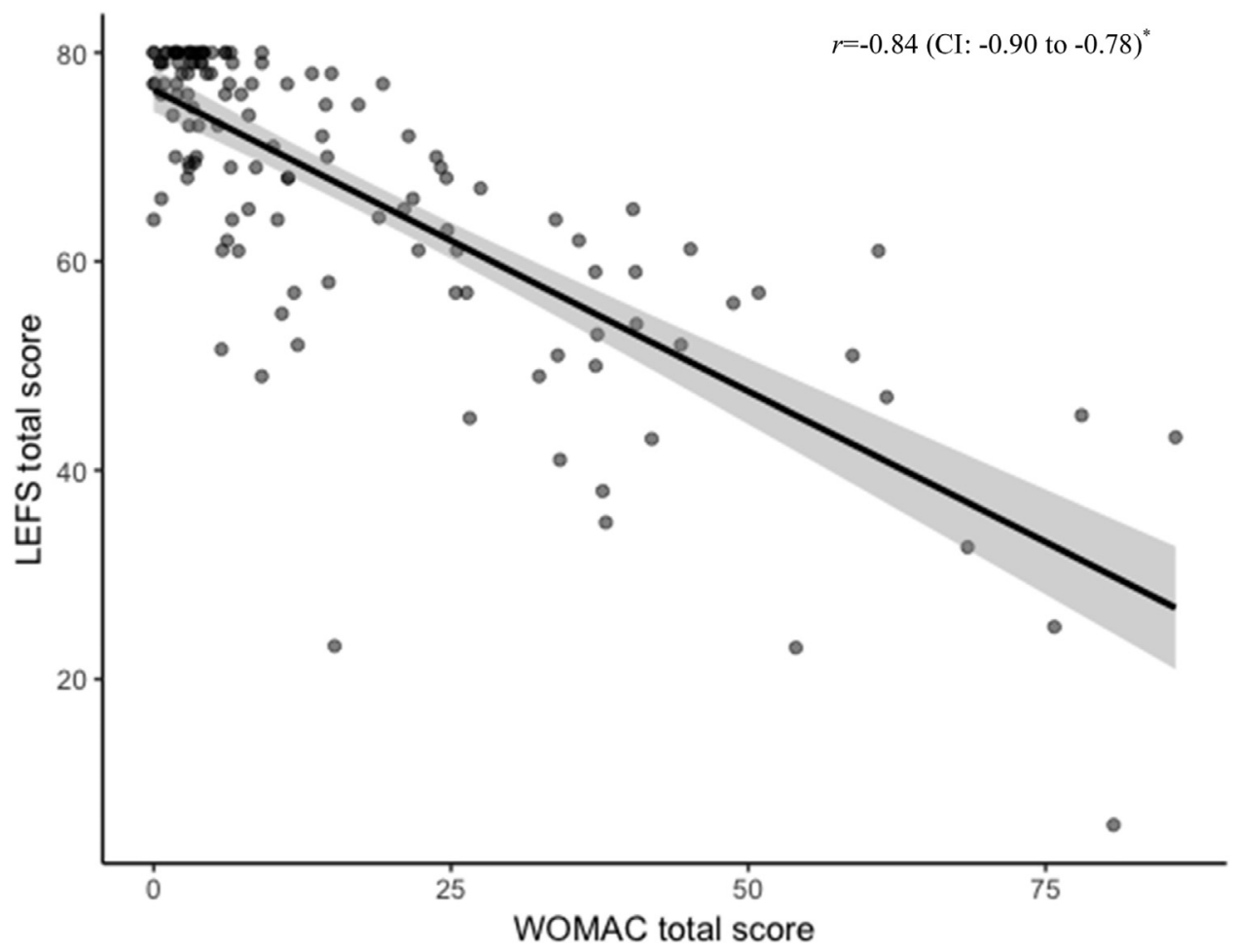

Fig. 3. Correlation between the WOMAC total score and the LEFS score. *Spearman correlation coefficient $(95 \% \mathrm{CI})$.

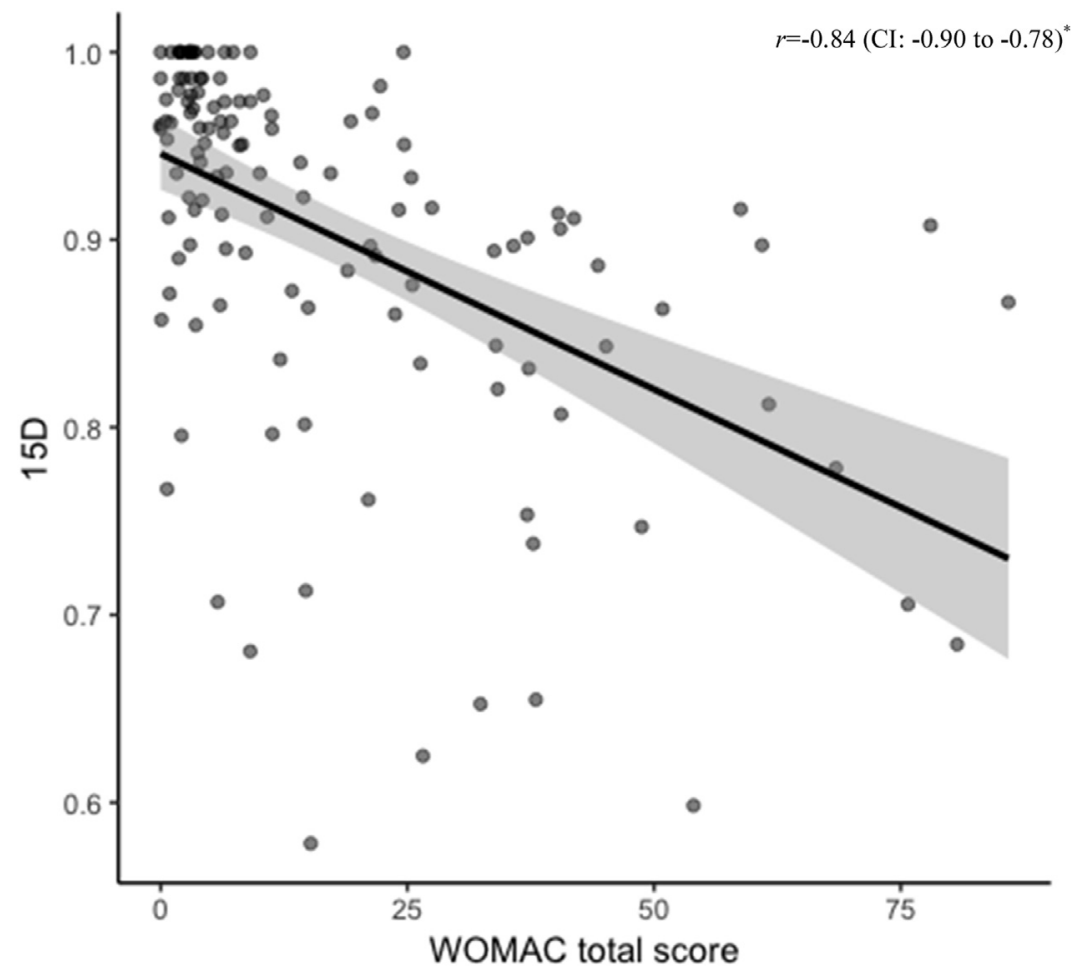

Fig. 4. Correlation between the WOMAC total score and the 15D score. *Spearman correlation coefficient $(95 \% \mathrm{CI})$. 


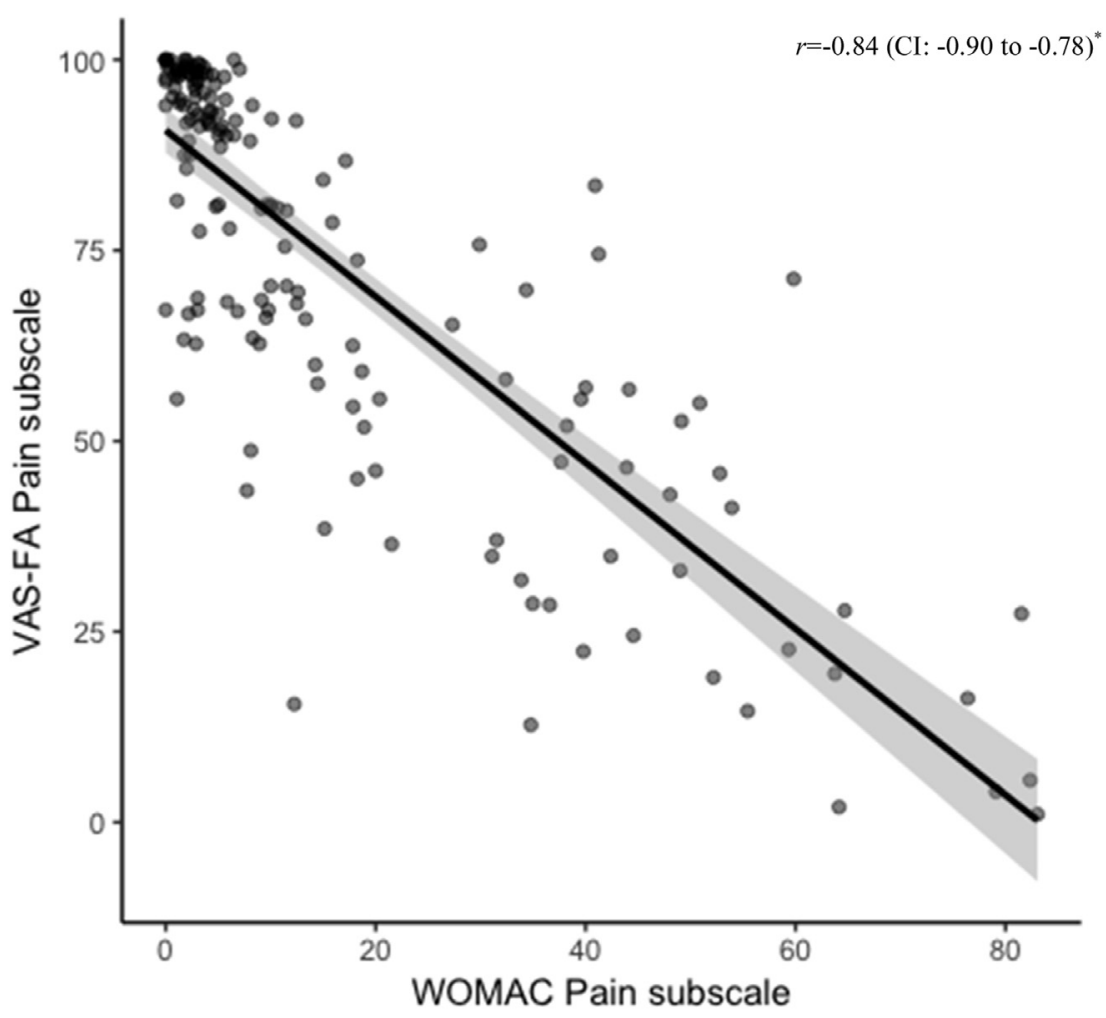

Fig. 5. Correlation between the WOMAC Pain subscale and the VAS-FA Pain subscale. *Spearman correlation coefficient $(95 \% \mathrm{CI})$.

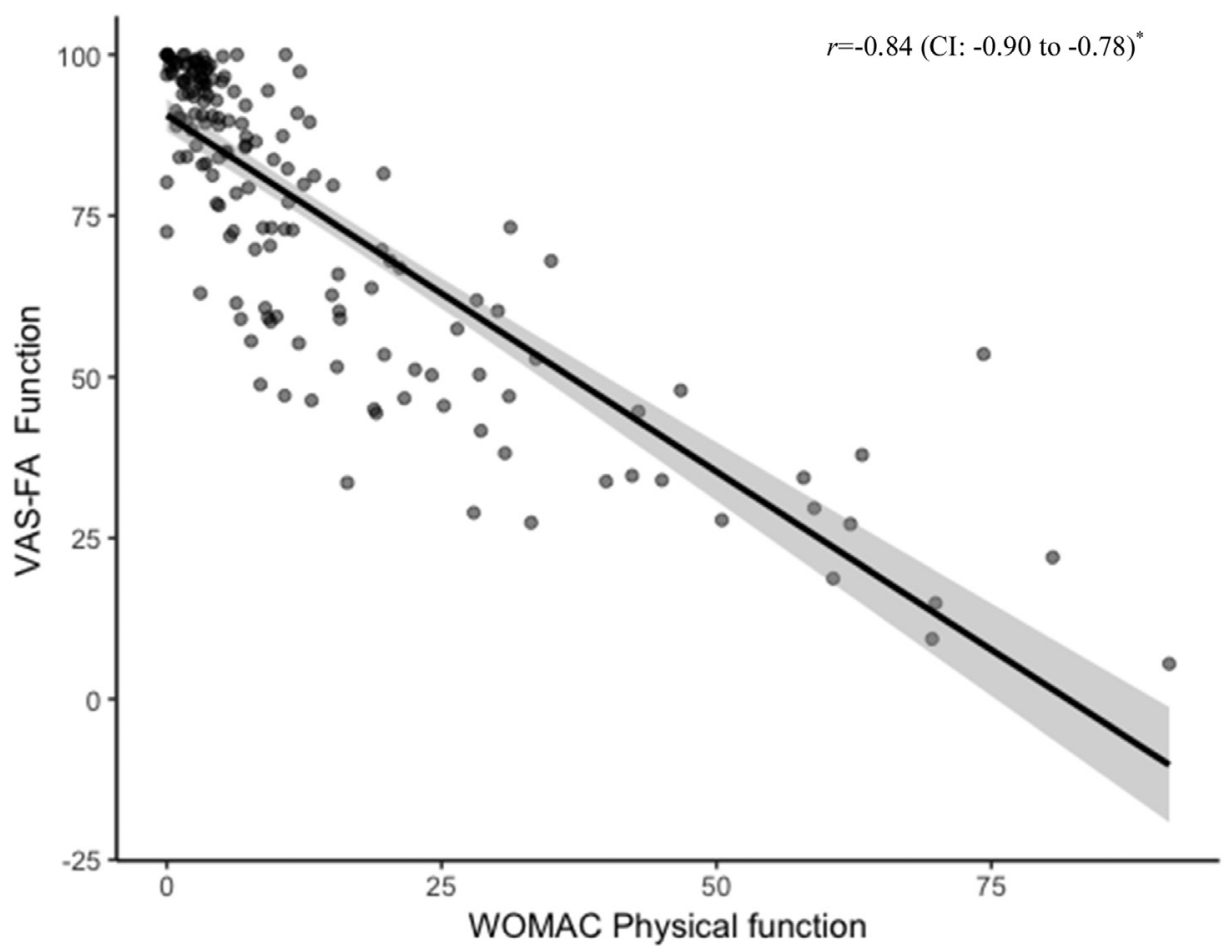

Fig. 6. Correlation between the WOMAC Physical Function subscale and the VAS-FA Function subscale. *Spearman correlation coefficient (95\% CI). 


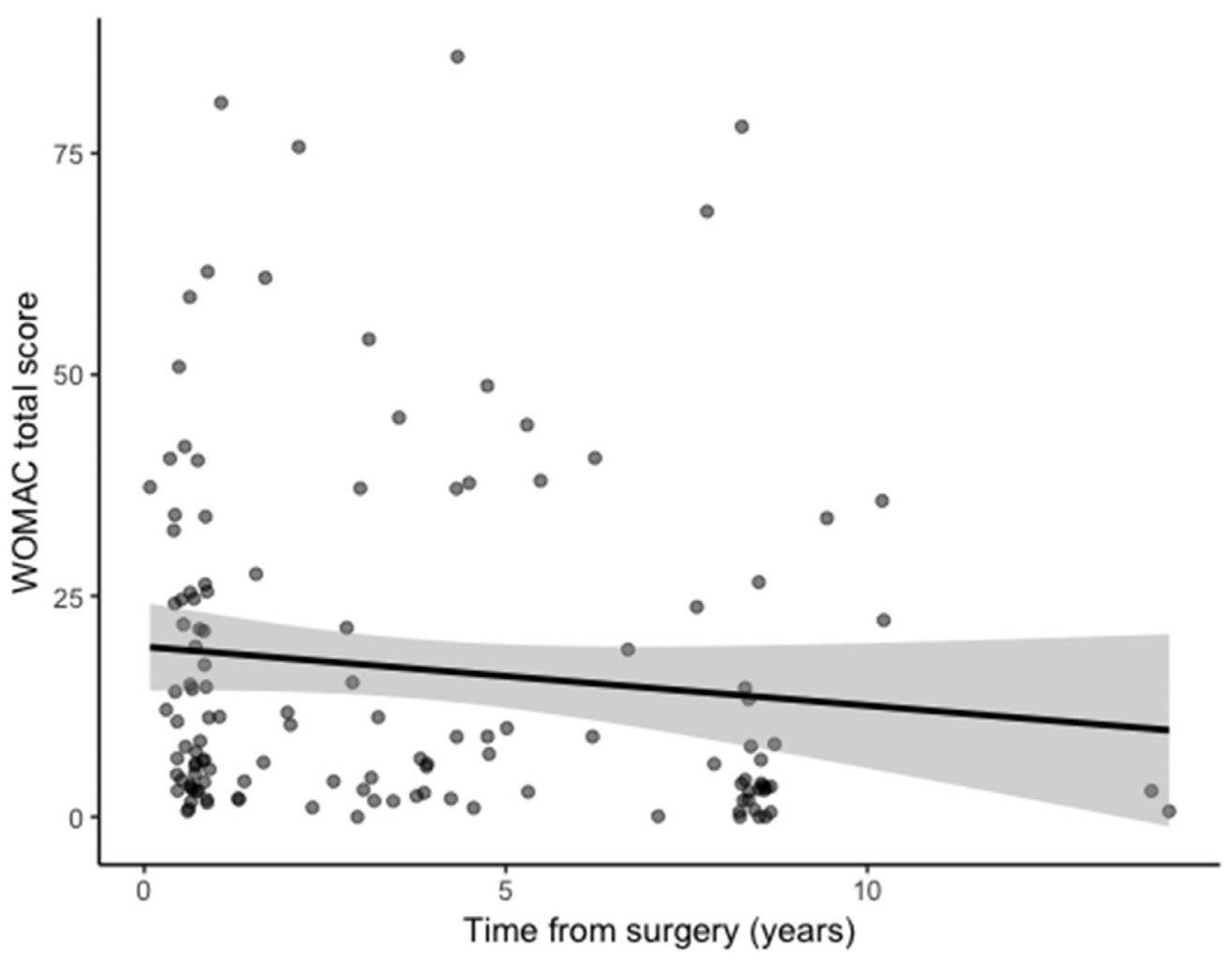

Fig. 7. Correlation between the WOMAC total score and time from surgery in years. *Spearman correlation coefficient $(95 \% \mathrm{CI})$.

VAS FA

LEFS

$15 \mathrm{D}$

\section{VAS General Health}

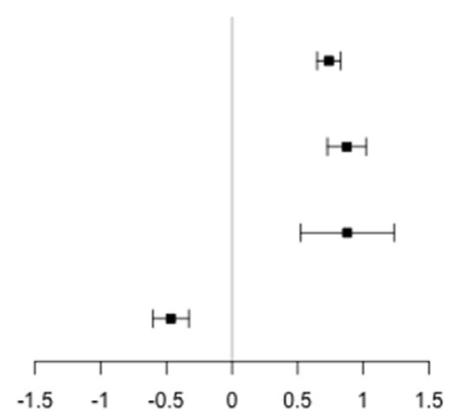

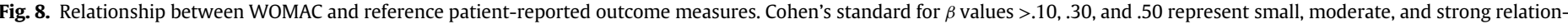

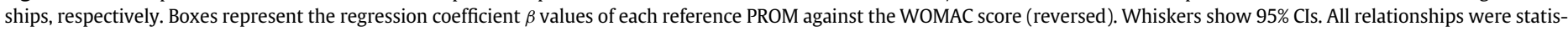
tically significant $(p<.001)$. Abbreviations: LEFS = Lower Extremity Functional Scale; VAS FA = Visual Analogue Scale Foot and Ankle.

poor reliability and good Cronbach's $\alpha(.80)$ in previous studies (17). This may be because the Stiffness subscale contains 2 items. The VAS scaled WOMAC was used in our study.

In the present study, high Cronbach's $\alpha$ values (>.90) indicate excellent internal consistency for the WOMAC. The Cronbach's $\alpha$ was lowest in the Stiffness subscale $(.86)$ yet still indicated very good internal consistency. The results are consistent with the previous validation study of the WOMAC (17). The correlations and relationships with other validated foot and ankle-specific PROMs were high, indicating strong validity in patients with foot and ankle problems. Because the VAS General Health is a concise 1-item instrument for assessing overall health, it is understandable that the relationship with the VAS general health was not as high as the foot-specific PROMs.

The strength of this study was the use of properly validated foot and ankle-specific PROMs. The WOMAC (17) and the LEFS (24) have been previously validated according to Rasch analysis (45), which offers many benefits compared with other validation methods (50). Because stiffness is a known problem after ankle fractures (51-55), it is important to take the stiffness into account when evaluating outcomes. However, our reference PROMs did not include such a subscale and thus may affect the results of the correlations. Nevertheless, the VAS-FA and LEFS are valid and reliable for foot and ankle surgery, and it is worthwhile to compare the WOMAC with these outcome measures. The long duration (mean 4 years) between surgery and completing the questionnaires can be considered a weakness. Nonetheless, the analyses of the present study also provide important information on how the WOMAC questionnaire functions years after surgery. The patients had high physical function and a low level of pain according to the reference outcome measures. This may be because they had had time to recover from the surgery. Despite the long interval from surgery, floor or ceiling effects were not confirmed, indicating that the WOMAC provided sufficient targeting and coverage for the present study population. Patients completed multiple questionnaires at once, which may be a concern regarding the study reliability. This study 
did not test the responsiveness of the WOMAC between multiple timepoints, which may also be considered a weakness.

In conclusion, this study provides support that the WOMAC yields valid scores for assessing the outcomes of pain, stiffness, and physical function after ankle fracture surgery. The results suggest that the WOMAC provides sufficient targeting and coverage even in long-term follow-up after surgery. Future prospective studies with a more heterogeneous population of patients having undergone foot and ankle surgery may provide further insight for the measurement properties of the WOMAC.

\section{References}

1. Hunt KJMD, Hurwit DBA. Use of patient-reported outcome measures in foot and ankle research. J Bone Joint Surg Am 2013;95:e118 (1-9).

2. Dawson J, Doll H, Fitzpatrick R, Jenkinson C, Carr AJ. The routine use of patient reported outcome measures in healthcare settings. BMJ (Clin Res) 2010;340:c186.

3. Marshall S, Haywood K, Fitzpatrick R. Impact of patient-reported outcome measures on routine practice: a structured review. J Eval Clin Pract 2006;12:559-568.

4. US Department of Health and Human Services FDA Center for Drug Evaluation and Research, US Department of Health and Human Services FDA Center for Biologics Evaluation and Research, US Department of Health and Human Services FDA Center for Devices and Radiological Health. Guidance for industry: patient-reported outcome measures: use in medical product development to support labeling claims: draft guidance. Health Qual Life Outcomes 2006;4:79.

5. SooHoo NF, Shuler M, Fleming LL. Evaluation of the validity of the AOFAS clinical rating systems by correlation to the SF-36. Foot Ankle Int 2003;24:50-55.

6. Button G, Pinney S. A meta-analysis of outcome rating scales in foot and ankle surgery: is there a valid, reliable, and responsive system? Foot Ankle Int 2004;25: 521-525.

7. Frost MH, Reeve BB, Liepa AM, Stauffer JW, Hays RD; Mayo/FDA Patient-Reported Outcomes Consensus Meeting Group. What is sufficient evidence for the reliability and validity of patient-reported outcome measures? Value Health 2007;10:S94S105.

8. Patrick DL, Burke LB, Powers JH, et al. Patient-reported outcomes to support medical product labeling claims: FDA perspective. Value Health 2007;10:S125-S137.

9. Ghandehari H, Mahabadi MA, Mahdavi SM, Shahsavaripour A, Seyed Tari HV, Safdari F. Evaluation of patient outcome and satisfaction after surgical treatment of adolescent idiopathic scoliosis using Scoliosis Research Society-30. Arch Bone Joint Surg 2015;3:109-113.

10. Doward LC, Gnanasakthy A, Baker MG. Patient reported outcomes: looking beyond the label claim. Health Qual Life Outcomes 2010;8:89.

11. Bellamy N, Buchanan WW, Goldsmith CH, Campbell J, Stitt LW. Validation study of WOMAC: a health status instrument for measuring clinically important patient relevant outcomes to antirheumatic drug therapy in patients with osteoarthritis of the hip or knee. J Rheumatol 1988;15:1833-1840.

12. Bellamy N. Validation study of WOMAC: a health status instrument for measuring clinically important patient-relevant outcomes following total hip or knee arthroplasty in osteoarthritis. J Orthop Rheumatol 1988;1:95-108.

13. McConnell S, Kolopack P, Davis AM. The Western Ontario and McMaster Universities Osteoarthritis Index (WOMAC): a review of its utility and measurement properties. Arthritis Rheumatism 2001;45:453-461.

14. Wolfe F, Kong SX. Rasch analysis of the Western Ontario McMaster questionnaire (WOMAC) in 2205 patients with osteoarthritis, rheumatoid arthritis, and fibromyalgia. Ann Rheum Dis 1999;58:563-568.

15. Jinks C, Jordan K, Croft P. Measuring the population impact of knee pain and disability with the Western Ontario and McMaster Universities Osteoarthritis Index (WOMAC). Pain 2002;100:55-64.

16. Kasari D. The effects of exercise and fitness on serum lipids in college women. In: Unpublished master's thesis, University of Montana; 1976.

17. Kersten P, White PJ, Tennant A. The Visual Analogue WOMAC 3.0 scale: internal validity and responsiveness of the VAS version. BMC Musculoskel Dis 2010;11. 80-80.

18. Soininen JV, Paavolainen PO, Gronblad MA, Kaapa EH. Validation study of a Finnish version of the Western Ontario and McMasters University Osteoarthritis Index. Hip Int 2008:18:108-111.

19. Koli J, Multanen J, Häkkinen A, Kiviranta I, Kujala U, Heinonen A. Reliability of the Finnish versions of WOMAC and KOOS forms for knee osteoarthritis. Physiotherapy 2011;97(suppl):D203-D204.

20. Gur G, Turgut E, Dilek B, Baltaci G, Bek N, Yakut Y. Validity and reliability of Visual Analog Scale Foot and Ankle: the Turkish version. J Foot Ankle Surg 2017;56: 1213-1217.

21. Angthong C, Chernchujit B, Suntharapa T, Harnroongroj T. Visual Analogue Scale Foot and Ankle: validity and reliability of Thai version of the new outcome score in subjective form. J Med Assoc Thailand 2011;94:952-957.

22. Repo JP, Tukiainen EJ, Roine RP, Kautiainen H, Lindahl J, Ilves O, Järvenpää S, Häkkinen A. Reliability and validity of the Finnish version of the Visual Analogue Scale Foot and Ankle (VAS-FA). Foot Ankle Surg 2018;24:474-480.

23. Richter M, Zech S, Geerling J, Frink M, Knobloch K, Krettek C. A new foot and ankle outcome score: questionnaire based, subjective, Visual-Analogue-Scale, validated and computerized. Foot Ankle Surg 2006;12:191-199.
24. Repo J, Tukiainen E, Roine R, Sampo M, Sandelin H, Häkkinen A. Rasch analysis of the lower extremity functional scale for foot and ankle patients. Disabil Rehabil 2018.

25. Binkley JM, Stratford PW, Lott SA, Riddle DL. The Lower Extremity Functional Scale (LEFS): scale development, measurement properties, and clinical application. North American Orthopaedic Rehabilitation Research Network. Phys Ther 1999;79:371383.

26. Repo JP, Tukiainen EJ, Roine RP, Ilves O, Järvenpää S, Häkkinen A. Reliability and validity of the Finnish version of the Lower Extremity Functional Scale (LEFS). Disabil Rehabil 2017;39:1228-1234.

27. Sintonen H. The 15D instrument of health-related quality of life: properties and applications. Ann Med 2001;33:328-336.

28. Stavem K. Reliability, validity and responsiveness of two multiattribute utility measures in patients with chronic obstructive pulmonary disease. Qual Life Res 1999;8:45-54.

29. Moock J, Kohlmann T. Comparing preference-based quality-of-life measures: results from rehabilitation patients with musculoskeletal, cardiovascular, or psychosomatic disorders. Qual Life Res 2008;17:485-495.

30. McHorney CA, Tarlov AR. Individual-patient monitoring in clinical practice: are available health status surveys adequate? Qual Life Res 1995;4:293-307.

31. Cronbach LJ. Coefficient alpha and the internal structure of tests. Psychometrika 1951;16:297-334.

32. Prinsen CAC, Mokkink LB, Bouter LM, Alonso J, Patrick DL, de Vet HCW, et al. COSMIN guideline for systematic reviews of patient-reported outcome measures. Qual Life Res 2018;27:1147-1157.

33. Mukaka MM. A guide to appropriate use of correlation coefficient in medical research. Malawi Med J 2012:24:69-71.

34. Von Elm E, Altman DG, Egger M, Alonso J, Stratford PW, Knol DL, Bouter LM, de Vet HC. The Strengthening the Reporting of Observational Studies in Epidemiology (STROBE) statement: guidelines for reporting observational studies. PLoS Med 2007:4:e296.

35. Mokkink LB, Terwee CB, Patrick DL, Alonso J, Stratford PW, Knol DL, Bouter LM, de Vet HC. The COSMIN checklist for assessing the methodological quality of studies on measurement properties of health status measurement instruments: an international Delphi study. Qual Life Res 2010;19:539-549.

36. Domsic RT, Saltzman CL. Ankle osteoarthritis scale. Foot Ankle Int 1998;19:466-471.

37. Pinsker E, Daniels TR, Inrig T, Warmington K, Beaton DE. The ability of outcome questionnaires to capture patient concerns following ankle reconstruction. Foot Ankle Int 2013;34:65-74.

38. Kitaoka HB, Meeker JE, Phisitkul P, Adams SB Jr., Kaplan JR, Wagner E. AOFAS position statement regarding patient-reported outcome measures. Foot Ankle Int 2018. 1071100718809066

39. Shazadeh Safavi P, Janney C, Jupiter D, Kunzler D, Bui R, Panchbhavi VK. A systematic review of the outcome evaluation tools for the foot and ankle. Foot Ankle Specialist 2018. 1938640018803747.

40. Turchin DC, Schemitsch EH, McKee MD, Waddell JP. Do foot injuries significantly affect the functional outcome of multiply injured patients? J Orthop Trauma 1999; $13: 1-4$.

41. Milner SA, Davis TRC, Muir KR, Greenwood DC, Doherty M. Long-term outcome after tibial shaft fracture: is malunion important? JBJS 2002;84:971-980.

42. Martin RL, Irrgang JJ, Lalonde KA, Conti S. Current Concepts Review: Foot and ankle outcome instruments. Foot Ankle Int 2006;27:383-390.

43. Stiegelmar R, McKee MD, Waddell JP, Schemitsch EH. Outcome of foot injuries in multiply injured patients. Orthop Clin N Am 2001;32:193-204.

44. Pinsker E, Inrig T, Daniels TR, Warmington K, Beaton DE. Reliability and validity of 6 measures of pain, function, and disability for ankle arthroplasty and arthrodesis. Foot Ankle Int 2015;36:617-625.

45. Rasch G. Studies in Mathematical Psychology: I. Probabilistic Models for Some Intelligence and Attainment Tests. Nielsen \& Lydiche, Oxford, England; 1960.

46. Davis A, Badley E, Beaton D, Kopec J, Wright JG, Young NL, Williams JI. Rasch analysis of the Western Ontario McMaster (WOMAC) osteoarthritis index: results from community and arthroplasty samples. J Clin Epidemiol 2003;56: 1076-1083.

47. Roorda LD, Jones CA, Waltz M, Lankhorst GJ, Bouter LM, van der Eijken JW, Willems WJ, Heyligers IC, Voaklander DC, Kelly KD, Suarez-Almazor ME. Satisfactory cross cultural equivalence of the Dutch WOMAC in patients with hip osteoarthritis waiting for arthroplasty. Ann Rheum Dis 2004;63:36-42.

48. Rothenfluh D, Reedwisch D, Müller U, Ganz R, Tennant A, Leunig M. Construct validity of a 12-item WOMAC for assessment of femoro-acetabular impingement and osteoarthritis of the hip. Osteoarthritis Cartilage 2008;16:1032-1038.

49. Ryser L, Wright BD, Aeschlimann A, Mariacher-Gehler S, Stucki G. A new look at the Western Ontario and McMaster Universities Osteoarthritis Index using Rasch analysis. Arthritis Care Res 1999;12:331-335.

50. Panayides P, Robinson C, Tymms P. The assessment revolution that has passed England by: Rasch measurement. Br Educ Res J 2010;36:611-626.

51. Chesworth BM, Vandervoort AA. Comparison of passive stiffness variables and range of motion in uninvolved and involved ankle joints of patients following ankle fractures. Physical Ther 1995;75:253-261.

52. Brodie I, Denham R. The treatment of unstable ankle fractures. J Bone Joint Surg $\mathrm{Br}$ 1974;56:256-262.

53. Ahl T, Dalén N, Holmberg S, Selvik G. Early weight bearing of displaced ankle fractures. Acta Orthop Scand 1987:58:535-538.

54. Egol KA, Tejwani NC, Walsh MG, Capla EL, Koval KJ. Predictors of short-term functional outcome following ankle fracture surgery. JBJS 2006;88:974-979.

55. Hancock MJ, Herbert RD, Stewart M. Prediction of outcome after ankle fracture. J Orthop Sports Phys Ther 2005;35:786-792. 